\title{
Mimicking the Secretory Action of a Gland by a Composite System Made of a pH-Responsive Surfactant-Based Hydrogel and a Dialysis Membrane
}

\author{
Alessio Cesaretti, ${ }^{\dagger}$ Irene Di Guida, ${ }^{\dagger}$ Naishka E. Caldero-Rodríguez, ${ }^{\dagger,+}$ Catia Clementi, ${ }^{\dagger}$ \\ Raimondo Germani, ${ }^{\dagger}$ and Pier Luigi Gentili*, ${ }^{\dagger}$ \\ ${ }^{\dagger}$ Department of Chemistry, Biology and Biotechnology, University of Perugia, Via Elce di sotto 8, Perugia 06123, Italy \\ ${ }^{\ddagger}$ Department of Chemistry, College of Natural Sciences, University of Puerto Rico, Río Piedras Campus, San Juan 00931-3346, \\ Puerto Rico
}

Supporting Information

ABSTRACT: The hydrogel obtained by a zwitterionic Noxide surfactant is proposed as the core of a $\mathrm{pH}$-responsive artificial gland model. The viscosity and $\mathrm{pH}$ variations, induced by pulse additions of acid and base, are investigated by a pyridinium salt and alizarin red $S$, respectively. The artificial gland model is implemented by enclosing the gel within a dialysis membrane, and its secretory action is tested by monitoring the release of a fluorescent acridinium salt.

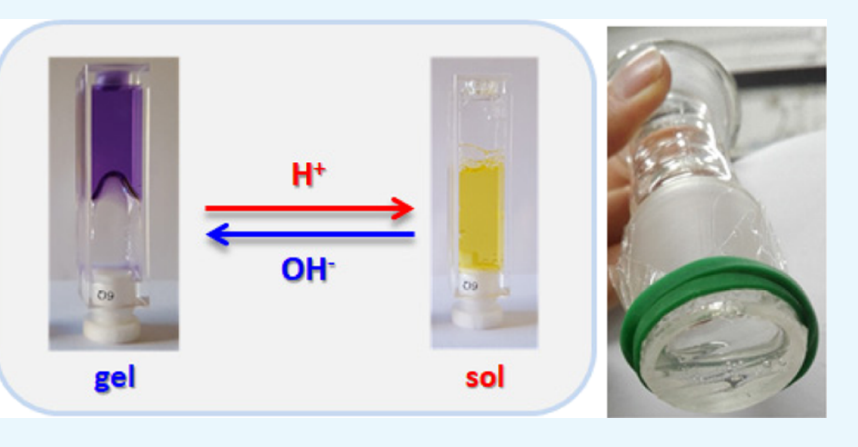

miniaturized and implanted in living beings to interplay with cells or organelles for biomedical applications.

After proposing molecular sensors ${ }^{12-14}$ and artificial neural models, ${ }^{15-17}$ in this work, we present a surfactant-based hydrogel, combined with a dialysis membrane (DM), as a $\mathrm{pH}$ sensitive secreting gland model. In the human effector system, glands are sac-like tissue structures, which play vital roles in the physiology of many organs by secreting either hormones or fluids. Glands exert their secretory action in response to excitatory inputs coming from the brain; these inputs can be either periodic or occasional. We show that it is possible to mimic the secretory action of a gland by using the $\mathrm{pH}$-sensitive gel generated by the amphoteric $p$-dodecyloxybenzyldimethylamine N-oxide (pDoAO) surfactant (see Chart 1) as the core and a $\mathbf{D M}$ as the boundary. The composite system is indicated as pDoAO + DM, hereinafter. On the one hand, an acidic input promotes the gel-to-sol transition; on the other hand, a basic input restores the gel state. We use the fluorescent $N$ octyl-acridinium perchlorate $\left(\mathbf{C}_{8} \mathbf{A c}\right)$ as a hormone-like compound for the pDoAO + DM artificial gland model. To monitor the $\mathrm{pH}$-induced gel-sol transitions and the properties of the gel and sol phases of pDoAO, we have chosen two compounds as follows: alizarin red $\mathrm{S}$ (ARS) as the $\mathrm{pH}$ probe and 2,4-(diphenylamino)phenyl-ethenyl-1-methylpyridinium iodide (PyI) as the viscosity probe (Chart 1 ).

Received: October 25, 2018

Accepted: November 26, 2018

Published: December 6, 2018 
Chart 1. Molecular structures of the zwitterionic surfactant pDoAO, the probes ARS and the pyridinium salt (PyI), and the acridinium salt $\left(\mathrm{C}_{8} \mathrm{Ac}\right)$

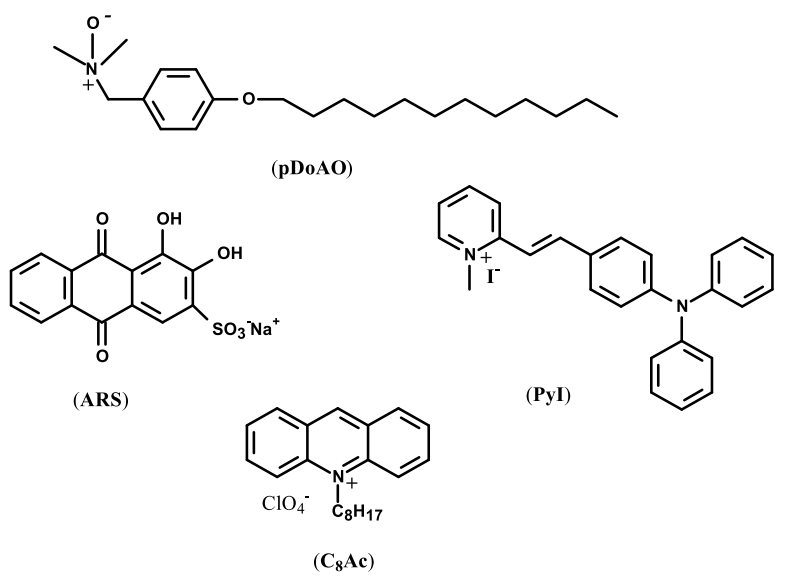

\section{RESULTS AND DISCUSSION}

The amphoteric pDoAO surfactant has $\mathrm{p} K_{\mathrm{a}}=4.9$ in water. ${ }^{18}$ When the $\mathrm{pH}$ of the solution is neutral or alkaline, the pDoAO molecules are zwitterionic and they carry no net charges. Under these conditions, previous structural investigations ${ }^{18,19}$ revealed that when the concentration of $\mathrm{pDoAO}$ is larger than the critical micellar value $\left(\mathrm{cmc}=1.6 \times 10^{-5} \mathrm{M}\right)$, pDoAO molecules spontaneously self-assemble into long, flexible wormlike micelles that are interconnected in dynamic network structures because of the weak repulsion between the neutral headgroups and the adhesive action of the hydrocarbon tails. At acidic $\mathrm{pH}<\mathrm{pK}$, the single pDoAO molecules become positively charged, thus affecting the supramolecular structure of the micelles. In fact, it has been shown ${ }^{18,19}$ that the wormlike micelles become shorter and their networks become less entangled and viscous.

The ARS salt is a dye whose UV-visible absorption spectrum depends appreciably on the $\mathrm{pH}$ of the solution (see Figure 1A). The two hydroxyl groups (see Chart 1) have two distinct $\mathrm{p} K_{\mathrm{a} i}$ values, which are $5.5\left(\mathrm{p} K_{\mathrm{a} 1}\right)$ and $11\left(\mathrm{p} K_{\mathrm{a} 2}\right)$, respectively. ${ }^{20}$ At $\mathrm{pH} \approx 3, \mathrm{ARS}$ is in its monoanionic form (see Chart 1) and its absorption spectrum, in the visible region, is characterized just by one band centered at $421 \mathrm{~nm}$, conferring a yellow color to its solution. At $\mathrm{pH} \approx 7.5$, ARS is mainly in its dianionic form and its absorption spectrum in the visible region consists of a broad band centered at $495 \mathrm{~nm}$, conferring a magenta color. In Milli-Q water, at $\mathrm{pH} \approx 5.5$, the ARS spectrum is a convolution of the contribution of the bands at 421 and $495 \mathrm{~nm}$ because its solution contains a mixture of the mono- and dianionic forms. When ARS is dissolved in water, buffered at $\mathrm{pH}=7.5$, at a concentration of $8.0 \times 10^{-5} \mathrm{M}$ and in the presence of $\mathbf{p D o A O}$ micelles, in a ratio [micelles]/[ARS] $\approx 15$, the characteristic band of dianionic ARS is red-shifted from 495 to $540 \mathrm{~nm}$ (giving a purple color) and it is slightly less intense (see the last spectrum in Figure 1B showing the titration of ARS with pDoAO; Table S1 in the Supporting Information contains the details of the additions). This spectral modification suggests that the dianionic ARS molecules interplay with the micellar aggregates, which have the surfactants in their zwitterionic state, and the equilibrium association constant has been estimated to be $K=550$ (see eq. S1 and Figure S1 in the Supporting Information). When ARS is dissolved in water, buffered at $\mathrm{pH}=3.1$, at a concentration
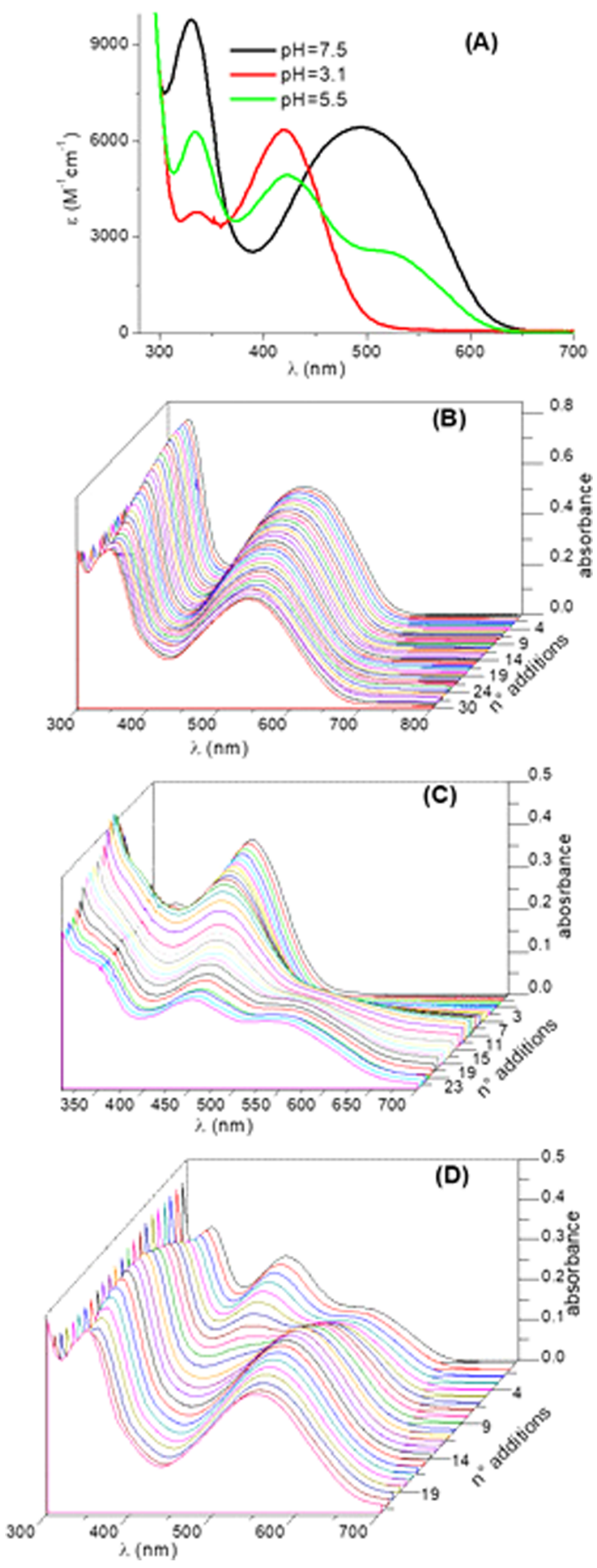

Figure 1. Absorption spectra of ARS in water at different $\mathrm{pHs}$ in (A); titration of ARS with pDoAO at $\mathrm{pH}=7.5$ in (B), at $\mathrm{pH}=3.1$ in (C), and in pure water in (D). The plotted spectra have been corrected by considering the dilution due to each addition of the surfactant solution. The values of the ratio $[\mathrm{pDoAO}] /[\mathrm{ARS}]$, at every addition, are reported in the Supporting Information.

of $5.8 \times 10^{-5} \mathrm{M}$ and in the presence of $\mathrm{pDoAO}$ micelles, in a ratio $[$ micelles $] /[$ ARS $] \approx 11$, the band of the monoanionic form red-shifts and wanes in favor of the appearance of a spectral contribution in the region of dianionic ARS (see the last spectrum in Figure 1C showing the titration of ARS with pDoAO; Table S2 contains the details of the additions). The spectral evolution in Figure $1 \mathrm{C}$ reveals that the monoanionic 
ARS molecules interplay with the micelles, consisting of both protonated and zwitterionic surfactants, and they are presumably shielded by the micelles, wherein they partially deprotonate. The association constant between ARS and the micelles has been estimated to be $K=4700$ (see Figure S2). When ARS is dissolved in pure unbuffered water at $5.4 \times 10^{-5}$ $M$ and in the presence of pDoAO micelles, in a ratio $[$ micelles $] /[$ ARS $] \approx 9$, the band of the monoanionic form, at $421 \mathrm{~nm}$, wanes in favor of the red-shifted band of the dianionic form, at $540 \mathrm{~nm}$ (see the last spectrum in Figure 1D showing the titration of ARS with pDoAO; Table S3 contains the details of the additions). This spectral evidence confirms that the zwitterionic pDoAO surfactants deprotonate the monoanionic ARS and the resulting dianionic ARS molecules interplay with the micelles through an equilibrium association constant $K=4600$ close to that estimated at $\mathrm{pH}=3.1$ (see Figure S3).

When the concentration of pDoAO in pure water is increased from $\approx 5 \times 10^{-4}$ to $7 \times 10^{-3} \mathrm{M}$, the solution zeroshear viscosity rises more than 10 times, from $\eta \approx 1 \mathrm{cP}$ up to $\eta$ $\approx 11 \mathrm{cP}$. The increase in [pDoAO] makes the network of wormlike micelles so entangled that the solution becomes viscoelastic. ${ }^{18}$ When $\left[\right.$ pDoAO] $\approx 10^{-1} \mathrm{M}$, the system becomes a firm gel, having $\eta \approx 2900 \mathrm{cP}$. It is possible to probe this viscosity growth by using the push-pull PyI compound (see Chart 1). PyI is both a polarity and a viscosity probe because of its mechanism of relaxation after photoexcitation. Its relaxation pathway involves a first fluorescent Franck-Condon excited state, which can relax to a structural twisted chargetransfer state emitting at lower energies. ${ }^{21}$ The polarity of the microenvironment affects the shape, position, and intensity of PyI fluorescence emission, ${ }^{21}$ whereas the viscosity mainly affects intensity. In fact, the stronger the viscosity, the higher the PyI fluorescence quantum yield, and a linear relation between $\Phi_{\mathrm{F}}(\mathrm{PyI})$ and $\log (\eta)$ has been found (see the data in Figure S4). The tighter the pDoAO micelle web, the more hindered the PyI structural rearrangement and the higher the probability of PyI emission. The viscosities of both the viscoelastic solution (when $[\mathrm{pDoAO}] \approx 10^{-2} \mathrm{M}$ ) and the gel (when $[\mathrm{pDoAO}] \approx 10^{-1} \mathrm{M}$ ) are $\mathrm{pH}$-sensitive. Figure 2 shows the effect of consecutive injections of acid and base in a viscoelastic pDoAO solution (see also Figure S5 in Supporting

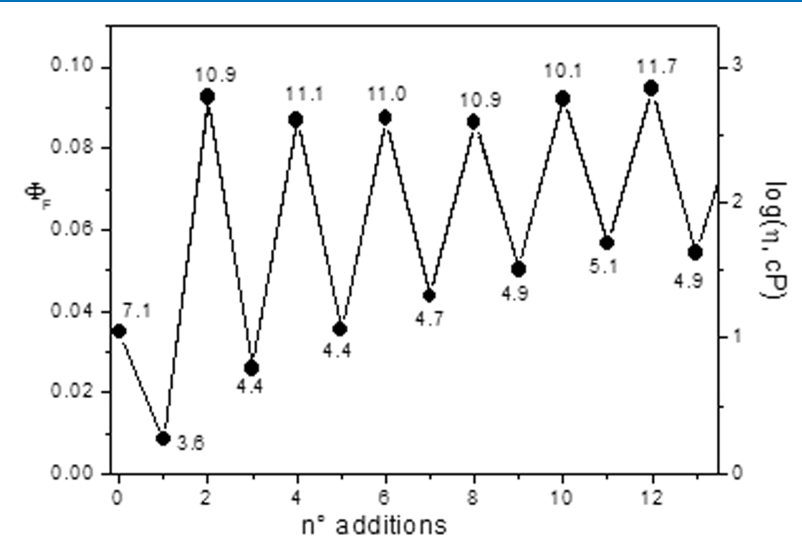

Figure 2. Effect of acid $(\mathrm{HCOOH})$ and base $(\mathrm{NaOH})$ additions on $\Phi_{\mathrm{F}}$ of PyI $\left(2 \times 10^{-5} \mathrm{M}\right)$ and the logarithm of viscosities $(\eta$ in $\mathrm{cP})$ of the viscoelastic solutions containing $[\mathrm{pDoAO}] \approx 10^{-2} \mathrm{M}$. The values close to the dots in the graph correspond to the $\mathrm{pH}$ of the solution after every addition.
Information, for the analytical details of the single injections). Before any addition, the system is characterized by the following parameters: $\mathrm{pH} \approx 7, \Phi_{\mathrm{F}}(\mathbf{P y I}) \approx 0.035$, and $\eta \approx 11$ cP. After the first addition of acid, both $\mathrm{pH}$ and $\Phi_{\mathrm{F}}(\mathbf{P y I})$ as well as $\eta$ decrease appreciably. The addition of acid protonates the oxygen atoms of the $\mathrm{N}^{+}-\mathrm{O}^{-}$groups, and the surfactant molecules, becoming positively charged, repel mutually. The ultimate micro-heterogeneous effect of acid addition is a shattered web of small rodlike micelles (according to the previous structural investigations ${ }^{18,19}$ ), exhibiting a reduced viscosity with respect to that of the original entangled web. After neutralizing the acid by injection of an equimolar amount of $\mathrm{NaOH}$, both $\mathrm{pH}$ and $\Phi_{\mathrm{F}}(\mathbf{P y I})$ as well as $\eta$ jump well beyond the initial values, recorded before any addition of acid and base. The high $\mathrm{pH}(\approx 11)$, achieved by the addition of $\mathrm{NaOH}$, guarantees the deprotonation of all of the $\mathrm{N}^{+}-\mathrm{OH}$ groups, which become zwitterionic. The threads of wormlike micelles can self-assemble tightly without suffering electrostatic repulsions. The resulting network shows the highest $\Phi_{\mathrm{F}}(\mathbf{P y I})$ $(\approx 0.09)$ and the strongest viscosity $(\eta \approx 620 \mathrm{cP})$. Further sequential injections of acid and base reproduce the consecutive drop and growth of $\mathrm{pH}, \Phi_{\mathrm{F}}(\mathbf{P y I})$, and $\eta$. However, the extent of the $\mathrm{pH}, \Phi_{\mathrm{F}}(\mathbf{P y I})$, and $\eta$ variations $[\Delta \mathrm{pH}$, $\Delta \Phi_{\mathrm{F}}(\mathrm{PyI})$, and $\left.\Delta(\log (\eta))\right]$ tends to shrink monotonically in the first consecutive injections. After eight additions of acid and base, $\Delta \mathrm{pH}, \Delta \Phi_{\mathrm{F}}(\mathbf{P y I})$, and $\Delta(\log (\eta))$ stabilize to 5, 0.04, and 1.2, respectively, except for random fluctuations. The initial shrinking of $\Delta \mathrm{pH}, \Delta \Phi_{\mathrm{F}}(\mathbf{P y I})$, and $\Delta(\log (\eta))$ is ascribable to a sort of ageing effect in the acidified solution of pDoAO. This ageing effect is reproducible and detectable even by injecting amounts of acid and base different from those used to obtain the results shown in Figure 2 (see, for instance, Figure S6). Of course, the extent of $\Delta \mathrm{pH}, \Delta \Phi_{\mathrm{F}}(\mathbf{P y I})$, and $\Delta(\log (\eta))$ depends on how much acid and base is added every time. They are maximized when acid and base are added in excess with respect to the moles of pDoAO. Moreover, the shrinking of $\Delta \mathrm{pH}, \Delta \Phi_{\mathrm{F}}(\mathbf{P y I})$, and $\Delta(\log (\eta))$ is particularly pronounced if the anion of the acid interplays strongly with the $\mathrm{N}^{+}-\mathrm{OH}$, for example, if the acid is $\mathrm{HCl}$ (see Figure S7). The cause of the ageing effect is the accumulation of ionic salts (such as $\mathrm{Na}^{+} \mathrm{HCOO}^{-}$or $\mathrm{Na}^{+} \mathrm{Cl}^{-}$) in the solution of cationic surfactant molecules, which screens repulsive electrostatic interaction and induces the formation of a network of threadlike micelles accompanied by viscosity increase. ${ }^{18}$

When $[\mathrm{pDoAO}] \approx 10^{-1} \mathrm{M}$, the solution is a gel with $\mathrm{pH} \geq$ 7 and $\eta \approx 2900 \mathrm{cP}$. The $\mathrm{pH}$ of the gel is probed by ARS whose spectrum (see Figure 3C) exhibits the characteristic band of the dianionic form embedded within the pDoAO micelle network (the maximum of the absorption band is at $565 \mathrm{~nm}$ ) and looks purple. The viscosity of the gel is probed by PyI, whose $\Phi_{\mathrm{F}}$ is 0.17 . By adding an equimolar amount of $\mathrm{CH}_{3} \mathrm{SO}_{3} \mathrm{H}$, the $\mathrm{pH}$ and the viscosity drop. In fact, the system becomes an acidified sol. The acidity is probed by ARS whose absorption spectrum shows the characteristic band of the monoanionic form, centered at $434 \mathrm{~nm}$, giving a yellow color. The reduction in viscosity is visible by naked eye (see Figure $3 \mathrm{~A}, \mathrm{~B})$ and is quantitatively monitored through the fluorescence quantum yield of PyI, which is equal to 0.13 and corresponds to $\eta \approx 510 \mathrm{cP}$, that is, almost six times lower than the viscosity of the initial gel. By neutralizing the acid, the $\mathrm{pH}$ jumps to alkaline values, as confirmed by the absorption spectrum of ARS, which red-shifts and confers a purple color to the system (see Figure 3C,D). Moreover, the solution again becomes a 

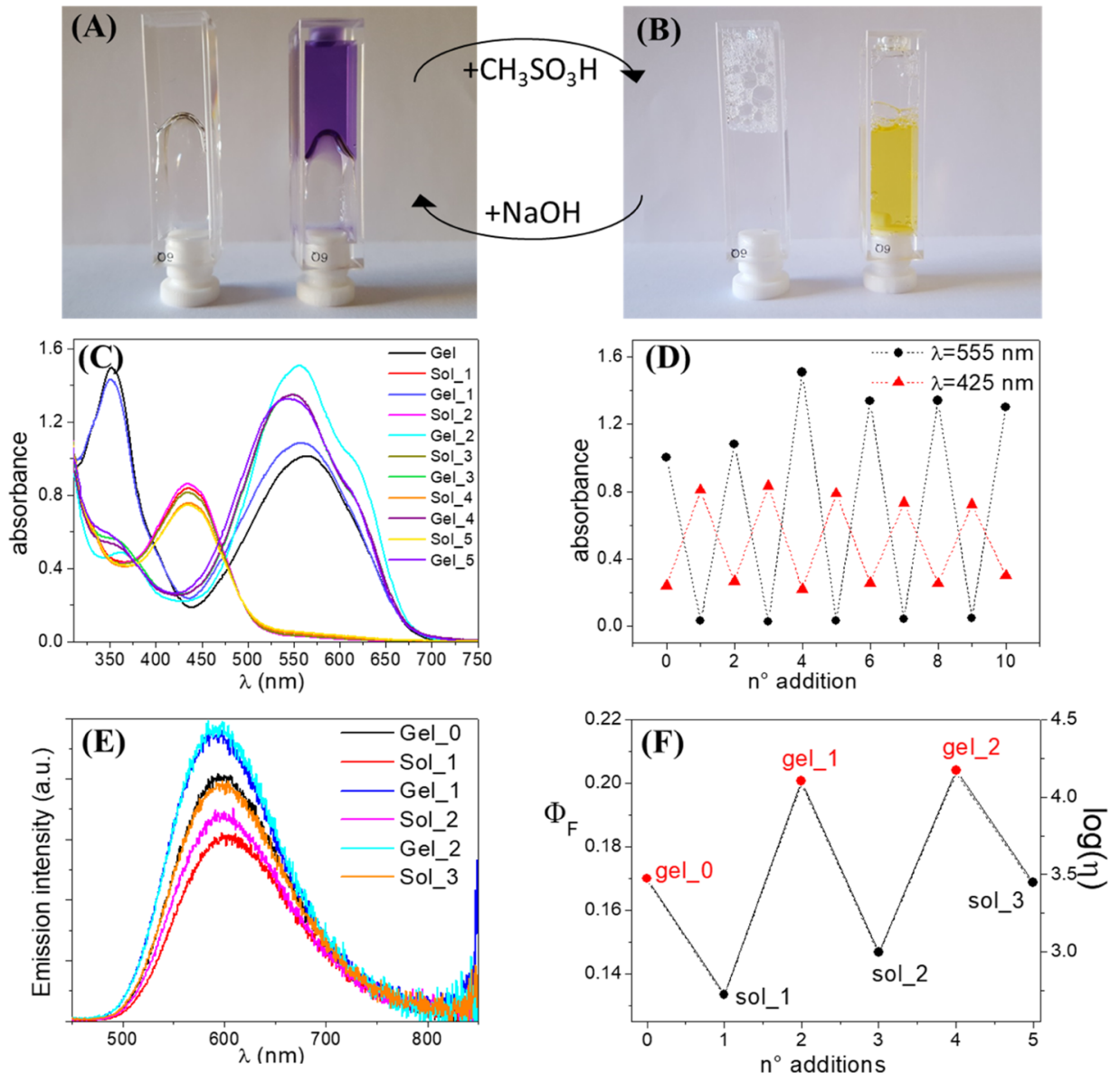

Figure 3. Gel (in A) to sol (in B) and reverse transition induced by the addition of $\mathrm{CH}_{3} \mathrm{SO}_{3} \mathrm{H}$ and $\mathrm{NaOH}$, respectively, in the absence (left cuvette) and presence (right cuvette) of ARS. Spectral modification of ARS (in C,D) after the sequential injections of $\mathrm{CH}_{3} \mathrm{SO}_{3} \mathrm{H}$ and $\mathrm{NaOH}$. Intensity changes in the emission spectrum of PyI (in E) and changes in $\Phi_{\mathrm{F}}(\mathrm{PyI})$ and $\log (\eta)$ of the solution containing $[\mathrm{pDoAO}] \approx 10^{-1} \mathrm{M}($ in $\mathrm{F})$ due to sequential injections of equimolar amounts of $\mathrm{CH}_{3} \mathrm{SO}_{3} \mathrm{H}$ and $\mathrm{NaOH}$.

gel, but with a much stronger viscosity: $\eta \approx 12200 \mathrm{cP}$, as probed by $\Phi_{\mathrm{F}}(\mathrm{PyI})$ that becomes 0.20 (see Figure 3E,F). This high viscosity is due to the surfactant molecules, which are now all in their zwitterionic form and show a very rigid network of entangled wormlike micelles. The probes, ARS and PyI, are in such small concentrations with respect to that of pDoAO that they do not affect the properties of the sol and gel phases. By injecting cyclically and repeatedly the acid and the base, the system with $[\mathrm{pDoAO}] \approx 10^{-1} \mathrm{M}$ shows an ageing effect similar to that found in the case of viscoelastic solutions with [pDoAO $\approx \approx 10^{-2} \mathrm{M}$ (see Figure $3 \mathrm{~F}$ ). After the shrinking stage, the viscosity change associated with the gel-sol transition stabilizes and its excursion ranges between $\approx 14000 \mathrm{cP}$ (gel state) and $\approx 2700 \mathrm{cP}$ (sol state), as probed by $\Phi_{\mathrm{F}}(\mathbf{P y I})$ that varies from $\approx 0.20$ to $\approx 0.17$. The $\eta$ values are 2 orders of magnitude larger than those spanned by the viscoelastic fluid shown in Figure 2 , where the viscosity ranges between $\approx 690$ and $\approx 40 \mathrm{cP}$, as soon as the ageing stage is completed.

The $\mathrm{pH}$ responsiveness of the zwitterionic pDoAO-based gel that has been presented in this work can be thus exploited to mimic the secretory action of a gland. The gel is segregated from a collecting aqueous solution through a DM. Figure 4 shows pictures of the device. The activity of the artificial gland model $(\mathbf{p D o A O}+\mathbf{D M})$ is tested by monitoring the secretion of the fluorescent probe $\mathrm{C}_{8} \mathrm{Ac}$ that interplays with $\mathrm{pDoAO}$ through its positive charge and its long alkyl chain. The release
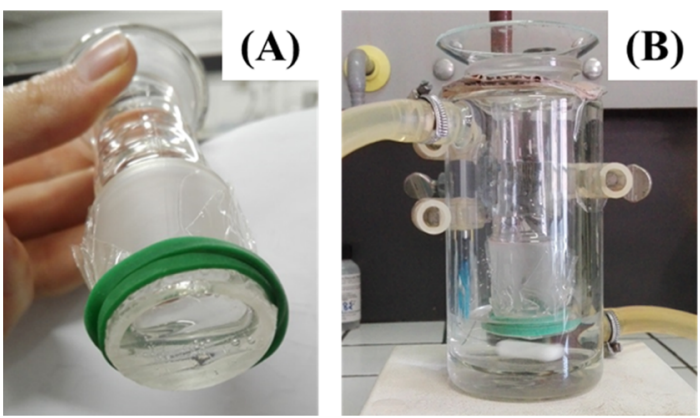

Figure 4. Structure of the artificial gland model. A glass tube closed at the bottom by the DM (A) and segregating the solution of the surfactant in water. The bottom part of the glass tube is immersed in water that is thermostated and under stirring within a beaker (B).

of pDoAO molecules is also monitored at regular time intervals (see the Experimental Section and paragraph I.3 of the Supporting Information for the details).

The results are shown in Figure 5. Figure 5A reports the molar fraction of $\mathbf{C}_{8} \mathbf{A c}$ secreted by the gel (squared red points) and the sol (circled black points) over time (determined fluorometrically). There is a significant difference between the two phases because in the case of the gel, the trend of $\chi_{\left(\mathrm{C}_{8} \mathrm{Ac}\right)}$ versus time reaches a plateau at $0.030 \pm 0.005$, whereas in the case of the sol, it grows monotonically up to a complete 

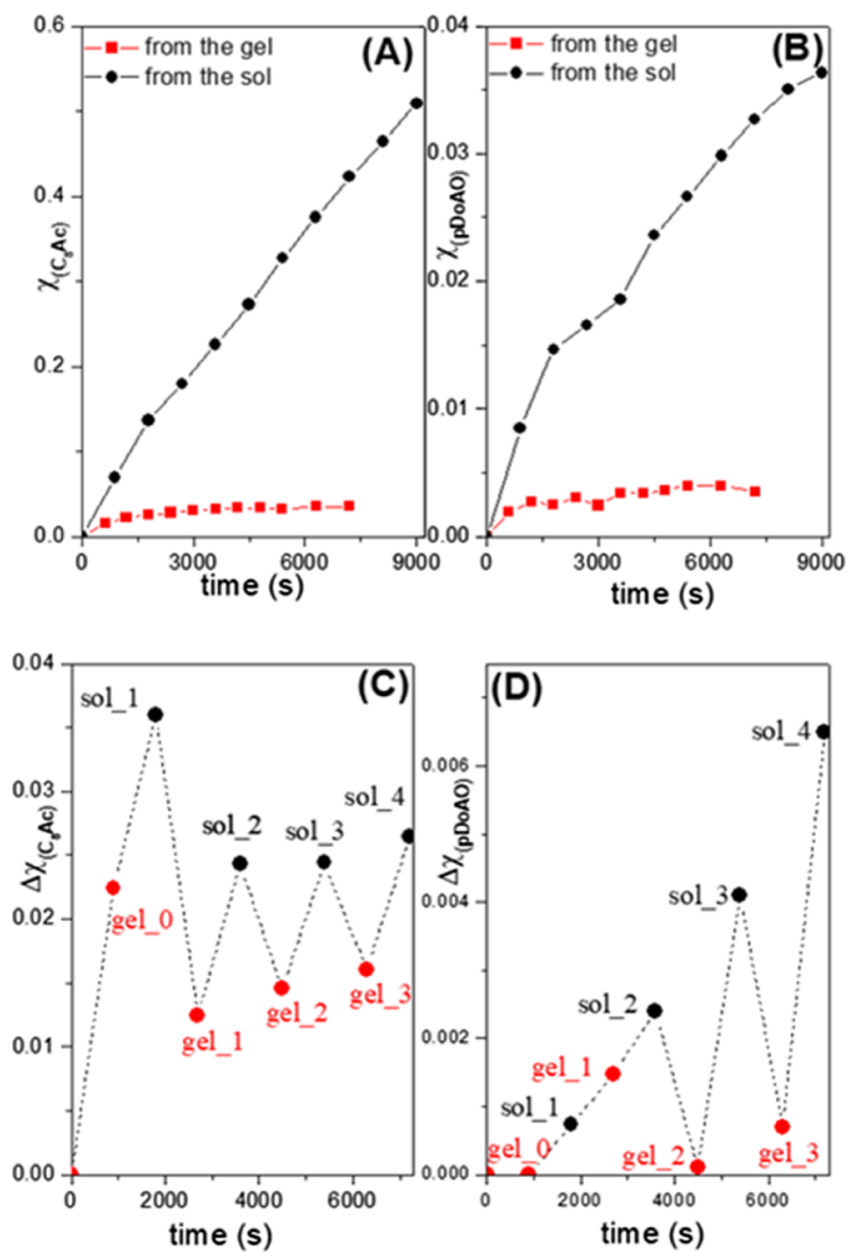

Figure 5. Trends of $\chi_{\left(\mathrm{C}_{8} \mathrm{Ac}\right)}$ (in $\left.\mathrm{A}\right)$ and $\chi_{(\mathrm{pDoAO})}$ (in B) released over time by the artificial gland model $($ pDoAO + DM) in the gel (red squares) and sol (black circles) states, respectively. Trends of the molar fraction increments $\Delta \chi_{\left(\mathrm{C}_{8} \mathrm{Ac}\right)}$ (in C) and $\Delta \chi_{(\mathrm{pDoAO})}$ (in D) secreted by pDoAO + DM after sequential injections of $\mathrm{CH}_{3} \mathrm{SO}_{3} \mathrm{H}$ and $\mathrm{NaOH}$, respectively. For the concentrations used, see the Supporting Information.

secretion of $\mathrm{C}_{8} \mathrm{Ac}\left(\chi_{\left(\mathrm{C}_{8} \mathrm{Ac}\right)}=1.0 \pm 0.1\right)$ in about $13 \mathrm{~h}$. The different secretory activities of pDoAO + DM in its sol and gel states are due not only to the distinct viscosities of the two phases but also to the contribution of DM. In fact, as shown in paragraph I.4 of the Supporting Information, DM releases $\mathrm{C}_{8} \mathrm{Ac}$ more easily from an acidic than from an alkaline aqueous solution. Figure 5B shows the release of the surfactant over time (determined spectrophotometrically). The behavior of the two phases is sharply different. From the gel phase, only $\chi_{\text {(pDoAO })}=0.0025 \pm 0.0005$ is lost in $2 \mathrm{~h}$, and after reaching the plateau, $\chi_{(\mathrm{pDoAO})}$ does not grow over time. From the sol phase, a molar fraction of $\chi_{(\mathrm{pDoAO})}=(0.036 \pm 0.010)$ is released in 2 $\mathrm{h}$, but it does not stop when the collecting solution is pure water. The composite system pDoAO + DM can be a promising artificial gland model if it also responds to periodic inputs. By injecting equimolar amounts of $\mathrm{CH}_{3} \mathrm{SO}_{3} \mathrm{H}$ and $\mathrm{NaOH}$ every $15 \mathrm{~min}$ (see the Supporting Information for the details), pDoAO + DM switches reversibly from the gel to the sol phase and back. Figure 5C shows the consecutive increments of $\chi_{\left(\mathrm{C}_{8} \mathrm{Ac}\right)}\left(\Delta \chi_{\left(\mathrm{C}_{8} \mathrm{Ac}\right)}\right)$ in the collecting aqueous solution. Figure $5 \mathrm{D}$ shows those of $\chi_{(\mathrm{pDoAO})}\left(\Delta \chi_{(\mathrm{pDoAO})}\right)$. The trend of Figure $5 \mathrm{C}$ confirms that the secretory power of the sol state is always larger than that of the gel state. Moreover, after the first injections of $\mathrm{CH}_{3} \mathrm{SO}_{3} \mathrm{H}$ and $\mathrm{NaOH}$, pDoAO + DM exhibits an ageing effect analogous to that reported in Figure $3 \mathrm{~F}$. In fact, the viscosities of both the gel and the sol phases increase and the amounts of secreted $\mathbf{C}_{8} \mathbf{A c}$ decrease. Finally, Figure 5D confirms that the release of the surfactant is limited in the case of the gel state, whereas it is relentless in the case of the sol phase.

\section{CONCLUSIONS}

The composite pDoAO + DM system can play as a $\mathrm{pH}$ responsive gland model releasing positively charged and relatively small molecules, such as $\mathbf{C}_{8}$ Ac. Because pDoAO + DM loses portions of its surfactant molecules as well, it can be an artificial model of an apocrine gland that has the peculiarity of releasing part of its cytoplasm in its secretions. To the best of our knowledge, our composite pDoAO + DM system is the first example of an artificial apocrine gland model devised through nonbiological systems. Its core, made of pDoAO, offers, as other previously investigated hydrogels based on smart wormlike micelles, ${ }^{22}$ two advantages over alternative $\mathrm{pH}$ responsive polymer gels: ${ }^{23-25}$ first, the instantaneous response to $\Delta \mathrm{pH}$ inputs and second, biocompatibility. ${ }^{26,27}$ The pDoAO hydrogel also exhibits a peculiar ageing phenomenon that can be exploited as a memory effect, which is an added value for smart materials. To extend the secretory capabilities of our artificial gland model, we are scrutinizing the permeability of other membranes. Finally, pDoAO + DM can be transformed into an autonomous, chemically powered, $\mathrm{pH}$-sensitive artificial gland model if it is integrated with a proper $\mathrm{pH}$ oscillatory chemical reaction.

\section{EXPERIMENTAL SECTION}

Materials. The zwitterionic surfactant pDoAO was synthesized according to the procedure described in ref 28 . ARS, which is the sodium salt of 9,10-dihydro-3,4-dihydroxy9,10-dioxo-2-antracenesulfonic acid (ARS) of analytic grade, was purchased from Carlo Erba and used without any further purification. The viscosity probe, 2,4-(diphenylamino)phenylethenyl-1-methylpyridinium iodide (PyI), was synthesized according to the procedure described in ref 21 . The probe $\mathrm{C}_{8} \mathrm{Ac}$ was synthesized by quaternization of acridine with octyliodide in acetonitrile; then, the perchlorate anion was introduced by ionic exchange with sodium perchlorate in methanol. ${ }^{29}$ To implement the artificial gland, the hydrogel made of pDoAO was embedded within a DM, $0.03 \mathrm{~mm}$ thick, made of cellulose with pores having an average diameter of 24 $\AA$. The thickness of the membrane was $0.3 \mathrm{~mm}$. The $\mathrm{pH}$ values of aqueous solutions at 3.1 and 7.5 were attained by Britton buffers prepared by mixing an acid solution $\left(\mathrm{H}_{3} \mathrm{BO}_{3} 0.04 \mathrm{M}\right.$, $\mathrm{H}_{3} \mathrm{PO}_{4} 0.04 \mathrm{M}$ and $\mathrm{CH}_{3} \mathrm{COOH} 0.04 \mathrm{M}$ ) with a $0.2 \mathrm{M}$ solution of $\mathrm{NaOH}$, adjusting the ionic strength of the final solution at 5 $\mathrm{mM}$.

Spectrophotometric Measurements. Steady-state absorption and emission spectra were recorded by using a PerkinElmer Lambda 800 spectrophotometer and a Horiba FluoroMax-4 spectrofluorimeter of HORIBA Scientific operated by FluorEssence, respectively. The latter gives back fluorescence emission spectra considering corrections for both the monochromator response and the detector sensitivity. Fluorescence quantum yields (experimental error of ca. 7\%) 
were determined from the emission spectra of samples whose absorbance at the excitation wavelength was lower than 0.1 . The absorbance was maintained smaller than 0.1 to have a linear relationship between the absorbance and the emission intensity and avoid self-absorption effects. Tetracene $\left(\Phi_{\mathrm{F}}=\right.$ 0.16 in air-equilibrated cyclohexane $)^{30}$ was used as the fluorometric standard for the determination of the fluorescence quantum yields.

Viscosity Measurements. The viscosities of the surfactant solutions were measured by using a Fungilab Expert L viscometer. The sample temperature was maintained constant by a glass-jacked beaker connected to a thermostatic bath. The viscosity measurements were obtained using a spindle attachment. Viscosity values are the average of three distinct measurements.

pH Measurements. The $\mathrm{pH}$ measurements were performed at room temperature by using an Orion SA $720 \mathrm{pH}$ meter equipped with a Metrohm AG 9101 Herisau combined glass electrode.

Determination of the Activity of the Artificial Gland Model. For the implementation of the artificial gland model, a specific weighted amount of the pDoAO gel (read paragraph I.3 for the details) was enclosed in a glass tube (having a diameter of $2.20 \mathrm{~cm}$ ) closed at the bottom by the DM adhering to the external glass wall through a rubber band (see Figure 4). This device was maintained within a beaker containing $20 \mathrm{~mL}$ of deionized water, thermostated at $298 \mathrm{~K}$, and under constant magnetic stirring (see Figure 4B). The DM, conditioned in water about $15 \mathrm{~h}$ before investigating the behavior of the artificial gland model, was wholly dipped in water. Specific amounts of the $\mathrm{CH}_{3} \mathrm{SO}_{3} \mathrm{H}$ and $\mathrm{NaOH}$ solutions were injected from the top of the glass tube to induce the gel-to-sol and the sol-to-gel transitions, respectively. To monitor the activity of the artificial gland model, we selected $\mathbf{C}_{8} \mathbf{A c}$ as a fluorescent probe. At regular time intervals, the absorption and emission spectra of $2 \mathrm{~mL}$ taken from the $20 \mathrm{~mL}$ water solution, collecting the molecules released by the artificial gland model, were recorded. The absorption spectra were useful to determine the amount of pDoAO molecules lost by the gland. The emission spectra were useful to calculate the amount of $\mathbf{C}_{8} \mathbf{A c}$ secreted by the gland. After $5 \mathrm{~min}$, time required to record the absorption and emission spectra, the 2 $\mathrm{mL}$ of water, used for the spectrophotometric analysis, was reintroduced within the collecting aqueous solution contained in the beaker. The quantitative determination of the secretory action of pDoAO in both the gel and the sol phase was reproduced at least twice.

\section{ASSOCIATED CONTENT}

\section{S Supporting Information}

The Supporting Information is available free of charge on the ACS Publications website at DOI: 10.1021/acsomega.8b02940.

Determination of association constants of ARS to pDoAO micelles; relation between $\Phi_{\mathrm{F}}(\mathrm{PyI})$ and $\eta$; effect of acid and base injections in $\eta$ of pDoAO viscoelastic fluids; activity of the artificial gland; and behavior of the DM (PDF)

\section{AUTHOR INFORMATION}

\section{Corresponding Author}

*E-mail: pierluigi.gentili@unipg.it (P.L.G.).

\section{ORCID}

Pier Luigi Gentili: 0000-0003-1092-9190

Notes

The authors declare no competing financial interest.

\section{ACKNOWLEDGMENTS}

Authors acknowledge the financial support by the University of Perugia (Fondo Ricerca di Base 2014, D. D. n. 170, 23/12/ 2014). N.E.C.-R. thanks the American Chemical Society IREU Program and the National Science Foundation for support under award number DMR-1262908. The authors thank Prof. C. Fortuna of the University of Catania for the synthesis of PyI.

\section{REFERENCES}

(1) Russell, S. J.; Norvig, P. Artificial Intelligence-A Modern Approach, 3rd ed.; Pearson Education Limited: Essex, U.K., 2016.

(2) Hagiya, M.; Konagaya, A.; Kobayashi, S.; Saito, H.; Murata, S. Molecular robots with sensors and intelligence. Acc. Chem. Res. 2014, 47, 1681-1690.

(3) Otero, T. F.; Martinez, J. G.; Arias-Pardilla, J. Biomimetic electrochemistry from conducting polymers. A review. Electrochim. Acta 2012, 84, 112-128.

(4) Hu, C.; Pané, S.; Nelson, B. J. Soft Micro- and Nanorobotics. Annu. Rev. Contr. Robot. Autonom. Syst. 2018, 1, 53-75.

(5) Grančič, P.; Štěpánek, F. "Chemical swarm robots". In Handbook of Collective Robotics-Fundamentals and Challenges; Kernbach, S., Ed.; Pan Stanford Publishing: Singapore, 2011.

(6) Hu, W.; Lum, G. Z.; Mastrangeli, M.; Sitti, M. Small-scale softbodied robot with multimodal locomotion. Nature 2018, 554, 81-85.

(7) Adamatzky, A.; de Lacy Costello, B.; Melhuish, C.; Ratcliffe, N. Experimental implementation of mobile robot taxis with onboard Belousov-Zhabotinsky chemical medium. Mater. Sci. Eng., C 2004, 24, 541-548.

(8) Hess, H.; Ross, J. L. Non-equilibrium assembly of microtubules: from molecules to autonomous chemical robots. Chem. Soc. Rev. 2017, 46, 5570-5587.

(9) Lund, K.; Manzo, A. J.; Dabby, N.; Michelotti, N.; JohnsonBuck, A.; Nangreave, J.; Taylor, S.; Pei, R.; Stojanovic, M. N.; Walter, N. G.; Winfree, E.; Yan, H. Molecular robots guided by prescriptive landscapes. Nature 2010, 465, 206-210.

(10) Kassem, S.; Lee, A. T. L.; Leigh, D. A.; Markevicius, A.; Solà, J. Pick-up, transport and release of a molecular cargo using a smallmolecule robotic arm. Nat. Chem. 2015, 8, 138-143.

(11) Tregubov, A. A.; Nikitin, P. I.; Nikitin, M. P. Advanced Smart Nanomaterials with Integrated Logic-Gating and Biocomputing: Dawn of Theranostic Nanorobots. Chem. Rev. 2018, 118, 1029410348.

(12) Gentili, P. L.; Rightler, A. L.; Heron, B. M.; Gabbutt, C. D. Extending human perception of electromagnetic radiation to the UV region through biologically inspired photochromic fuzzy logic (BIPFUL) systems. Chem. Commun. 2016, 52, 1474-1477.

(13) Gentili, P. L.; Rightler, A. L.; Heron, B. M.; Gabbutt, C. D. Discriminating between the UV-A, UV-B and UV-C regions by novel Biologically Inspired Photochromic Fuzzy Logic (BIPFUL) systems: A detailed comparative study. Dyes Pigm. 2016, 135, 169-176.

(14) Gentili, P. L. The fuzziness of a chromogenic spiroxazine. Dyes Pigm. 2014, 110, 235-248.

(15) Gentili, P. L.; Giubila, M. S.; Germani, R.; Romani, A.; Nicoziani, A.; Spalletti, A.; Heron, B. M. Optical Communication among Oscillatory Reactions and Photo-Excitable Systems: UV and Visible Radiation Can Synchronize Artificial Neuron Models. Angew. Chem., Int. Ed. 2017, 56, 7535-7540.

(16) Gentili, P. L.; Giubila, M. S.; Germani, R.; Heron, B. M. Photochromic and luminescent compounds as artificial neuron models. Dyes Pigm. 2018, 156, 149-159. 
(17) Gentili, P. L.; Horvath, V.; Vanag, V. K.; Epstein, I. R. Belousov-Zhabotinsky "Chemical Neuron" as a Binary and Fuzzy Logic Processor. Int. J. Unconv. Comput. 2012, 8, 177-192.

(18) Brinchi, L.; Germani, R.; Di Profio, P.; Marte, L.; Savelli, G.; Oda, R.; Berti, D. Viscoelastic solutions formed by worm-like micelles of amine oxide surfactant. J. Colloid Interface Sci. 2010, 346, 100-106.

(19) Goracci, L.; Germani, R.; Rathman, J. F.; Savelli, G. Anomalous behavior of amine oxide surfactants at the air/water interface. Langmuir 2007, 23, 10525-10532.

(20) Bishop, E. Indicators; Pergamon Press: Oxford, 1972.

(21) Carlotti, B.; Cesaretti, A.; Fortuna, C. G.; Spalletti, A.; Elisei, F. Experimental evidence of dual emission in a negatively solvatochromic push-pull pyridinium derivative. Phys. Chem. Chem. Phys. 2015, 17, $1877-1882$.

(22) Chu, Z.; Dreiss, C. A.; Feng, Y. Smart wormlike micelles. Chem. Soc. Rev. 2013, 42, 7174-7203.

(23) Ahn, S.-k.; Kasi, R. M.; Kim, S.-C.; Sharma, N.; Zhou, Y. Stimuli-responsive polymer gels. Soft Matter 2008, 4, 1151-1157.

(24) Qiu, Y.; Park, K. Environment-sensitive hydrogels for drug delivery. Adv. Drug Deliv. Rev. 2001, 53, 321-339.

(25) Koetting, M. C.; Peters, J. T.; Steichen, S. D.; Peppas, N. A. Stimulus-responsive hydrogels: Theory, modern advances, and applications. Mater. Sci. Eng., R 2015, 93, 1-49.

(26) García, M. T.; Campos, E.; Ribosa, I. Biodegradability and ecotoxicity of amine oxide based surfactants. Chemosphere 2007, 69, $1574-1578$.

(27) Singh, S. K.; Bajpai, M.; Tyagi, V. K. Amine Oxides: A Review. J. Oleo Sci. 2006, 55, 99-119.

(28) Goracci, L.; Germani, R.; Savelli, G.; Bassani, D. M. Hoechst 33258 as a $\mathrm{pH}$-sensitive probe to study the interaction of amine oxide surfactants with DNA. ChemBioChem 2004, 6, 197-203.

(29) Yamagishi, A.; Masui, T.; Watanabe, F. Selective activation of reactant molecules by reversed micelles. J. Phys. Chem. 1981, 85, 281285.

(30) Burgdorff, C.; Ehrhardt, S.; Loehmannsroeben, H. G. Photophysical properties of tetracene derivatives in solution. 2. Halogenated tetracene derivatives. J. Phys. Chem. 1991, 95, 42464249 . 